\title{
Degradación de residuos vegetales mediante inoculación con cepas microbianas
}

\section{(Vegetal waste degradation by microbial strains inoculation)}

\author{
Nubia Grijalva Vallejos ${ }^{1}$
}

\begin{abstract}
Resumen:
El tratamiento de los residuos vegetales producto de desechos urbanos, procesos agrícolas e industriales enfrenta varios problemas técnicos y constituye una preocupación ambiental importante. Entre ellos se destacan la permanencia de productos fitosanitarios en altas concentraciones en el material vegetal unido a la carencia de microorganismos que puedan tolerar dichos compuestos y logren descomponer eficientemente el sustrato. Las bacterias y principalmente los hongos de la podredumbre blanca son los mejores degradadores de materiales lignocelulósicos por su capacidad de sintetizar enzimas extracelulares hidrolíticas y oxidativas en altas cantidades. Trichoderma reesei, Aspergillus niger, Penicillium sp. y Phanerochaete chrysosporium son cepas modelo cuya eficiencia de degradación de materiales ricos en lignocelulosa incluso en presencia de contaminantes ha sido comprobada. Se están realizando varios estudios de mutagénesis dirigida, co-cultivo y expresión heteróloga, con el objeto de mejorar el contenido de enzimas (celulasas, xylanasas, y $\beta$-glucosidasas) en varias cepas, además de nuevas búsquedas genéticas para encontrar otros microorganismos con este potencial. Su principal aplicación es la producción industrial de etanol y metabolitos secundarios bajo condiciones controladas en procesos de fermentación. Esta revisión proporciona una perspectiva general de las estrategias y metodologías actualmente usadas para el aprovechamiento de residuos vegetales mediante inoculación de cepas microbianas.
\end{abstract}

Palabras clave: residuos, cepas, lignocelulosa, enzimas, inoculación.

\begin{abstract}
:
Vegetal waste treatment product of urban, agricultural and industrial processes has several technical problems and constitutes a significant environmental concern. Among them are the persistence of crop protection products in high concentrations in plant material and the lack of microorganisms that can tolerate such compounds and efficiently decompose the substrate. Bacteria and mainly white rot fungi are the main decomposers of lignin because of their ability to synthesize extracellular hydrolytic and oxidative enzymes in large quantities. Trichoderma reesei, Aspergillus niger, Penicillium sp. and Phanerochaete chrysosporium strains are model strains whose hight degradation efficiency with lignocellulose materials even in the presence of pollutants has been proven. Several studies such as directed mutagenesis, co-culturing and heterologous expression have been done in order to improve the content of some enzymes (cellulase, xylanase, and $\beta$-glucosidase) in model strains, additionally it has been done new genetic searches to find other microorganisms with this potential. Its main applications are the industrial production of ethanol and some seconday metabolites under controlled conditions in fermentation processes. This review provides an overview about strategies and methodologies currently used for vegetal waste utilization by inoculation of microbial strains.
\end{abstract}

Keywords: waste, strains, lignocellulose, enzymes, inoculation.

1 Universidad Tecnológica Equinoccial, Facultad de Ciencias de la Ingeniería, Quito - Ecuador (gvnj87974@ute.edu.ec) 


\section{Introducción}

La disponibilidad y abundancia de celulosa en nuestro entorno la convierten en materia prima muy atractiva para producir muchos artículos de importancia industrial y agronómica (Pratlma, Kalpana \& Avinash, 2012). La agricultura, la industria forestal, de producción de pulpa, papel y de alimentos, generan una gran cantidad de residuos ricos en lignina y celulosa que producen contaminación ambiental y además representan una pérdida por el desperdicio de materia que puede ser convertida en varios productos con valor agregado (Sánchez, 2008; Dashtban et al., 2010). Lastimosamente, a nivel global, muchos residuos celulósicos son generalmente eliminados mediante quema de biomasa (Pratlma et al., 2012). De igual forma, las agroindustrias producen una gran cantidad de basura y de contaminantes por lo cual es necesario que se optimicen sistemas de tratamientos para este tipo de residuos (Arvanitoyannis \& Varzakas, 2008).

La lignocelulosa es el principal componente de las células vegetales y consiste en tres elementos básicos: celulosa, hemicelulosa y lignina; es además la fuente de materia orgánica más abundante en la tierra (Pérez et al., 2002). Debido a su naturaleza recalcitrante, las plantas son naturalmente resistentes a la degradación microbiana y enzimática (Himmel et al., 2007), sin embargo la biodegradación de residuos vegetales ricos en lignocelulosa es un proceso importante para el ciclo del carbono en la naturaleza (Martinez et al., 2005).

La descomposición de la biomasa lignocelulósica involucra la formación de cadenas largas de polisacáridos y la subsecuente hidrólisis en cadenas de azúcares de 5 a 6 carbonos. En la producción de biocombustibles, estos azúcares pueden ser convertidos en bioetanol a través de un proceso de fermentación (Zhou et al., 2000). Con la ayuda de sistemas celulolíticos, la celulosa puede ser convertida a glucosa que es un producto multiútil mediante un proceso barato y biológicamente favorable (Pratlma et al., 2012).

El procesamiento consolidado de biomasa celulósica con microorganismos junto a la utilización combinada de sustratos permite la obtención de un producto final con propiedades adecuadas y se convierte en una alternativa de aprovechamiento con alto potencial (Lynd et al., 2005). Se ha reportado la actividad celulósica de muchos microorganismos en los que se incluyen varias cepas de hongos y bacterias tanto aeróbicas como anaeróbicas. Ente las especies de hongos responsables de la hidrólisis de biomasa celulósica se nombran Chaetomium, Fusarium, Myrothecium, Trichoderma, Penicillium, Aspergillus. Entre las especies bacterianas lignocelulolíticas se incluyen Trichonimpha, Clostridium, Actinomycetes, Bacteroides succinogenes, Butyrivibrio fibrisolveness, Ruminococcus albus, y Methanobrevibacter ruminantium (Pratlma et al., 2012)

Esta revisión se enfoca en la degradación de residuos vegetales mediante diferentes metodologías con la aplicación de inóculos microbianos. Además se hace un análisis de los 
principales problemas en su aplicación y de los últimos avances en la creación de cepas robustas para producción de enzimas y biocombustibles.

\section{Técnicas de tratamiento de residuos orgánicos}

Existen varias estrategias para el tratamiento de residuos agroindustriales, entre estas se destacan el compostaje y la fermentación en estado sólido.

El compostaje es un método muy usado en el reciclaje de basura municipal y agrícola (Chefetz et al., 1998). Consiste en una biotécnica de biodegradación aerobia en estado sólido en la cual se controlan el contenido de humedad, nutrientes, y ciertos parámetros físico - químicos establecidos para obtener como producto final compost que es usado como enmienda de suelos (Eweis et al., 1999) Las condiciones ambientales han sido un factor limitante en el desarrollo del compostaje ya que los procesos de degradación son lentos y variables según la estación lo que lleva a una calidad pobre del producto final (Benitez \& Gonzales, 2003). Existen muchas deficiencias en la implementación de procesos de compostaje como la falta de control de variables, tamaño y composición de la basura, uso indiscriminado de pesticidas en los cultivos así como la formación de gases tóxicos y líquidos (Lugo et al., 2005).

El uso exitoso del compost depende de su grado de madurez y estabilidad. La descomposición de compuestos fitotóxicos producidos en las fases tempranas del proceso de compostaje y la proporción de humus estable formado están influenciados por la naturaleza del material, su estructura y composición, y la capacidad de microorganismos para degradar las macromoléculas que constituyen el residuo (Vargas et al., 2007).

La fermentación en estado sólido (Solid state fermentation - SSF) es una alternativa actualmente utilizada para la producción de enzimas y otros metabolitos secundarios a partir de residuos agroindustriales en biorreactores que resulta poco costosa y requiere mínimas actividades de laboreo. Varias investigaciones con este sistema han obtenido buenos resultados en un amplio rango de aplicaciones tanto en escala de laboratorio, piloto e industrial (Lee, Darah, \& Ibrahim, 2010). En la investigación de Huang et al. (2010) se estudió mediante fermentación en estado sólido la degradación de residuos lignocelulósicos bajo estrés de plomo con la cepa fúngica Phanerochaete chrysosporium obteniéndose buenos resultados.

\section{Enzimas microbianas}

La degradación de la celulosa, fermentación, metanogénesis y reducción por sulfatos son procesos microbianos que coexisten en varios ambientes naturales y en procesos anaerobios inducidos en biorreactores (Pereira et al., 2010).

La degradación de la lignina y/o la modificación fúngica es un paso clave en la descomposición de la misma (Martinez et al., 2005). Existen varias especies de hongos que producen un gran número 
de enzimas en importantes cantidades y las liberan al ambiente sinérgicamente (Dashtban et al., 2009). Los hongos de la podredumbre blanca son los organismos más eficientes en la degradación de la celulosa, lignina y un amplio número de contaminantes persistentes como compuestos aromáticos clorados, hidrocarburos heterocíclicos aromáticos, algunos colorantes y polímeros sintéticos (Sánchez, 2008).

Las enzimas lignocelulolíticas son un grupo grande de proteínas extracelulares en las que se incluyen las enzimas ligninolíticas (peroxidasas y oxidasas) y enzimas hidrolíticas (pectinasas, hemicelulasas, quitinasas, celulasas, amilasas, esterasas, proteasas y manasas). Estas enzimas están presentes principalmente en cepas fúngicas (Godliving, 2012).

Una de las aplicaciones potenciales de las enzimas lignocelulolíticas en tecnologías ambientales es la producción de las mismas en grandes cantidades y a bajos costos. Una alternativa es usar nuevos organismos seleccionados con una síntesis notable de estas enzimas además de desarrollar estrategias para su sobreproducción (Elisashvili et al., 2008). Un número significativo de microorganismos han sido estudiados por su alto potencial en la degradación de la lignina (Dashtban et al., 2009; Huang et al., 2010; Li et al., 2008).

Las ligninasas, enzimas extracelulares que degradan la lignina, se clasifican en fenol oxidasas (lacasas) o heme peroxidasas [lignin peroxidasas (LiP), manganeso peroxidasa (MnP) y peroxidasa versátil (VP)] (Dashtban et al., 2010). Las lacasas son enzimas que juegan un rol muy importante en la detoxificación de residuos. La producción de estas enzimas es considerada una características de los hongos de las podredumbre blanca como P. chrysosporium (Martinez et al., 2005).

Un amplio rango de especies en las que se incluyen los ascomicetes, basidiomicetes y varias especies anaeróbicas producen enzimas lignocelulolíticas (Dashtan et al., 2009). Los hongos tienen dos tipos de sistemas enzimáticos extracelulares: el sistema hidrolítico, responsable de la degradación de polisacáridos, y el sistema lignolítico oxidativo y extracelular que degrada la lignina y abre los grupos fenilo. Las enzimas hidrolíticas más importantes son: exo-1-4- $\beta$-Dglucanasa, endo-1-4- $\beta$-D-glucanasa(EC 3.2.1.4), y xilanasa (EC 3.2.1.8) (Sánchez, 2008).

Las especies fúngicas como: Trichoderma, Penicillium, Aspergillus son capaces de producir grandes cantidades de celulasas y hemicelulasas extracelulares (Kumar \& Shweta., 2011). Trichoderma ressei y Aspergillus niger producen enzimas celulolíticas extracelulares como celulasas y xilanasas además secretan una amplia variedad de hemicelulasas en altas concentraciones (8 y 12 hemicelulasas, respectivamente) (Dashtan et al., 2009).

Las especies de Trichoderma son excelentes colonizadores de sus hábitats y son capaces de sobrevivir en diferentes ambientes. La utilización eficiente del sustrato y la capacidad de secreción de algunos metabolitos (antibióticos) y enzimas son algunas características importantes de este 
género (Schuster \& Schmoll, 2010). Trichoderma reesei y sus mutantes son los hongos ascomicetos más estudiados y son usados para la producción comercial de hemicelulasas y celulasas (Sánchez, 2008). La actividad celulolítica de T. ressei QM6a se ha mejorado mediante el uso de diferentes técnicas de mutagénesis (Dashtan et al., 2009).

Penicillium ochrochlorum Y5 ha sido recientemente aislado e identificado. Tiene una gran capacidad de degradar celulosa de paja de trigo, su actividad celulolítica es mayor que otras cepas (Yin et al., 2011).

$P$. chrysosporium, un holobasidiomicete, es una especie representativa y ampliamente estudiada por su habilidad de degradar un amplio rango de sustratos orgánicos y un amplio espectro de contaminantes químicos aromáticos, alicíclicos y alifáticos bajo condiciones de nutrientes limitantes (ligninolítico) y nutrientes suficientes (no lignolítico), incluso materiales bajo condiciones de estrés por plomo (Doddapaneni et al., 2005; Huang et al., 2010). Las cepas de $P$. chrysosporium degradan simultaneamente celulosa, hemicelulosa y lignina. La secuenciación del genoma de este hongo ha facilitado la caracterización de su proteoma extracelular completo (Sato et al., 2007).

En la última década se ha iniciado con la secuenciación del genoma de cepas fúngicas que ha dado origen a abundante información en este campo especialmente en la identificación de genes involucrados en la degradación de la lignocelulosa (Couturier et al., 2012). Existen ya proyectos de secuenciación del genoma de especies fúngicas que están en marcha, se incluyen 62 especies fúngicas en las que se encuentran 6 basidiomicetes y 27 ascomicetes (Dashtban et al., 2010).

Los hongos anaeróbicos producen un rango de enzimas celulolíticas y hemicelulolíticas en un complejo multienzimas (celulosomas). Estos hongos representan un grupo especial que habita el tracto gastro-intestinal de herbívoros rumiantes y muchos no rumiantes. En Piromyces sp. PC2, un hongo anaeróbico, se han aislado en el celulosoma 17 enzimas lignocelulolíticas para codificar genes incluyendo celulasas, $\beta$-glucoasidasas y hemicelulasas (Dashtan et al., 2009). Los celulosomas tienen muchas ventajas como la actividad sinérgica entre los componentes y una actividad hidrolítica en ambas (celulosa y hemicelulosas) pero los celulosomas fúngicos son menos caracterizados comparados a los celulosomas bacterianos (Ljungdahl, 2008).

Muchas cepas fúngicas no producen suficiente cantidad de una o más enzimas requeridas para la bioconversión de basura rica en lignocelulosa. La creación de cepas fúngicas robustas usando mutagénesis, expresión de genes heterólogos y cocultivo son estrategias importantes para mejorar la degradación de especies microbianas (Sharma \& Kuhad, 2010; Dashtan et al., 2009).

Los diferentes métodos de mutagénesis dirigida como mutagénesis por saturación, PCR propensa a errores y DNA por arrastramiento han sido usadas para mejorar las propiedades de enzimas específicas. Son buenos ejemplos los mutantes Penicillium verruculosum $28 \mathrm{~K}$ que tienen una 
mejora en la actividad celulasa y xilanasa mediante 4 ciclos de mutagénesis UV y mutantes de $T$. reseei (RUT-C30; T2C:T28C) que mejoran su actividad $\beta$-glucosidasa y endo-1,4- $\beta$-glucanasa II (Dashtan et al., 2009).

Algunos reportes indican que al mezclar varios aislados de microorganismos se da lugar a microfloras mucho más efectivas comparadas con cepas individuales de microorganismos (Kumar \& Shweta, 2011; Peng et al., 2010). Cultivos de mezclas fúngicas tienen muchas ventajas comparadas con sus monocultivos, en estas se incluyen la productividad, adaptabilidad y la utilización del sustrato. La principal desventaja de los cocultivos sin embargo es la complejidad de crecer múltiples microorganismos en el mismo cultivo (Lynd et al., 2002).

La expresión heteróloga de celulasas es una técnica actual usada para crear cepas fúngicas lignocelulolíticas vigorosas. Diferentes celulosas fúngicas con actividad específica y/o alta han sido clonadas y expresadas basadas en la necesidad para un sistema en un organismo. Hay importantes resultados en la expresión heteróloga de una $\beta$-glucosidase (ce/3a), colobiohidrolasa (I \& II), y una endoglucanasa usando un promotor fuerte $c b h 1$ termoestable en $T$. reseei (Dashtan et al., 2009).

La transformación genética de hongos degradadores de lignina es una herramienta importante actualmente disponible. Consiste en un sistema de transformación genética mediada por Agrobacterium para liberar el T - DNA, que lleva los genes que codifican para la $\beta$-glucuroidasa ( uiaA), proteína verde fluorescente ( $g f p$ ) y la fosfotransferasa higromicina ( $h p t$ ) al genoma nuclear de los hongos de la podredumbre blanca degradadores de lignina como son Phanerochaete chrysosporium, Pycnoporous cinnabariuns, entre otros (Sharma \& Kuhad, 2010).

La degradación sinérgica de la celulosa también puede ser llevada a cabo en sistemas de cultivos mixtos de bacterias celulolíticas y levaduras no celulolíticas, en las que las levaduras Saccharomyces cerevisiae utiliza los azúcares reducidos derivados de la degradación de la celulosa y los convierten en etanol (Pratlma et al., 2012)

En relación a las bacterias degradadoras de lignocelulosa, el grupo de investigación de Gupta et al. (2011) aisló ocho cepas de estos microorganismos a partir de 4 invertebrados diferentes: termita, caracol, oruga, gusano de libro. Determinaron una importante actividad celulasa y endoglucanasa. Las cepas seleccionadas fueron cultivadas junto a Saccharomyces cerevisiae y lograron producir etanol de una forma positiva.

En un estudio de Yang et al. (2009) se demostró que Anaerocellum thermophilum DSM 6725, una bacteria anaerobia, puede utilizar eficientemente varios tipos de biomasa vegetal no tratada así como celulosa cristalina y xilano. Los mecanismos de degradación todavía están por analizarse, y la importancia de este estudio radica en que existen pocos microorganismos cultivados que pueden degradar biomasa lignocelulósica sin un previo tratamiento químico. Los métodos 
anaeróbicos para la degradación de la materia orgánica son más eficientes aunque requieren tecnologías de alto nivel (Arvanitoyannis \& Varzakas, 2008).

\section{Inoculantes microbianos}

El uso de productos comerciales o inoculantes microbianos para acelerar el proceso de descomposición de residuos orgánicos es una estrategia actual y una de las mejores formas para generar subproductos de mejor calidad, sin embargo los resultados varían dependiendo del tipo de residuo tratado (Acevedo et al., 2005; Gaita \& Perez, 2007; Peng et al., 2010).

Los inoculantes son formulaciones de microorganismos cuya ventaja radica en la posibilidad de volver mucho más rápidos los procesos, usar escasa energía no renovable y ser una metodología limpia (no contaminantes del medio ambiente) (Benítez \& Gonzales, 2003).

En un estudio de compostaje realizado en China, la inoculación de desechos vegetales y tallos de flores con microorganismos lignocelulolíticos mejoró la biodegradación de los materiales pero no tuvo efectos significativos en el contenido de la mezcla, $\mathrm{pH}$ y contenido de carbono orgánico del compost final (Lu et al., 2004). En la aplicación de diferentes dosis de un inoculante termofílico bacteriano en compost a partir de residuos de rosas se obtuvo mejores resultados con una dosis baja $\left(30 \mathrm{ml} / \mathrm{m}^{3}\right)$. Factores como: el tamaño del residuo, el contenido de lignocelulosa y nutrientes de la pila, además de la posibilidad de la persistencia de productos fitosanitarios en la materia vegetal posiblemente fueron determinantes en el proceso ya que el tiempo de compostaje superó los 4 meses y se sugiere que podrían ser controlados para mejorar la calidad del compost final (Grijalva, 2007). Según un estudio realizado por Barrera \& Charry (2008) con un inoculante mixto formado por cinco cepas de microorganismos amilolíticos, este permitió acelerar el proceso en un principio, sin embargo las características finales fueron similares al control especialmente en el contenido de materia orgánica.

En un proceso de compostaje de residuos sólidos urbanos se logró disminuir notablemente el tiempo de tratamiento (4 semanas) al usar un inóculo en base a Bacillus subtilis, Pseudomonas fluorescens y Aspergillus fumigatus (Cariello et al., 2007)

La mayoría de los resultados de los estudios son positivos, sin embargo, existe un criterio conflictivo en la eficiencia del uso de estos inoculantes. Se destaca el hecho de que $<<$ la inoculación con ciertos microorganismos es efectiva bajo ciertas circunstancias>> (Adams 2007; Vargas et al., 2007).

\section{Problemas en la degradación de residuos}

El depósito de plaguicidas en una planta es eliminado de forma progresiva, con mayor o menor rapidez, según la acción de diversos factores: crecimiento del vegetal (aumento del peso), causas mecánicas y físicas (viento, lluvia; volatilización y solubilización) y degradación química 
(transformación de unas sustancias en otras) (Coscollá, 2008). Adicional a ello podría encontrarse la degradación biológica demostrada actualmente en varias investigaciones. La persistencia de un plaguicida en una planta depende de sus cualidades intrínsecas como estabilidad y polaridad además del tipo de sustrato vegetal, acciones mecánicas, físicas, entre otras. La cantidad de residuos presentes viene a su vez influida por varios factores que pueden agruparse en: factores propios de la aplicación, factores propios del cultivo y del medio ambiente, factores propios de la eliminación del producto (Oleas \& Fernández, 2001).

Casi todos los pesticidas persistentes usados en las plantas permanecen en ellas, son absorbidos por el suelo y se transportan por el agua superficial (González, 2007). En el caso de las flores ornamentales contienen altas concentraciones de productos de protección de cultivos (CPP) y contribuyen enormemente a la contaminación de la basura biológica, en este tipo de cultivo los cpp pueden alcanzar concentraciones mayores al $1 \mathrm{mg} / \mathrm{Kg}$ de residuo (Taube et al., 2002). Incluso se ha determinado la persistencia medioambiental de sustancias no utilizadas en la actualidad pero empleadas en el pasado.

Los pesticidas presentes en la basura orgánica deben ser degradados o mineralizados mediante tratamientos aerobios 0 anaerobios sin embargo bajo ciertas condiciones estos pueden ser persistentes a la degradación biológica (Taube et al., 2002). Existen varias rutas de remoción de sustancias tóxicas de un ambiente de compostaje en las que se incluyen la mineralización (completa biodegradación), biotransformación (deshalogenación), asimilación como nutriente en la biomasa microbiana, polimerización, volatilización, lixiviación y adsorción (Forgaty et al., 1991).

Los productos elaborados a partir de residuos biológicos son considerados ecológicos ya que son parte del proceso cerrado de reciclaje, sin embargo los pesticidas persistentes introducidos en este ciclo pueden aparecer en el producto final y afectar su potencial y calidad (Taube et al., 2002). Es necesario el desarrollo de métodos analíticos para la recuperación cuantitativa e identificación de la presencia de pesticidas en complejos de materiales ambientales residuales. Algunas vías de degradación dan lugar a metabolitos "dead-end" que pueden influir negativamente en la capacidad biodegradativa de los otros organismos presentes. Puede ser difícil separar la degradación microbiológica de los mecanismos de eliminación abióticos (adsorción, la conversión térmica, radiación, y volatilización) de plaguicidas, debe tomarse en cuenta que la volatilización puede representar una importante eliminación de pesticidas de material compostado, pero esto no necesariamente implica la destrucción de la molécula (Forgati et al., 1991).

Ante lo expuesto anteriormente es necesario estudiar por medio de ensayos el comportamiento de los plaguicidas en los residuos orgánicos (comportamiento post-tratamiento, curvas de degradación de los principales productos plaguicidas, etc.) (Breilh et al., 2005). 


\section{Comentarios concluyentes}

El potencial de los microorganismos como descomponedores de la materia orgánica, especialmente aquella rica en lignocelulosa, es actualmente un tema de estudio de gran importancia. El aprovechamiento de residuos como materia prima para la producción de biocombustibles, metabolitos secundarios de interés o enmiendas de suelos (humus, compost) es una necesidad actual tanto a nivel agrícola como industrial.

La diversidad microbiana en el planeta y la capacidad adaptativa de ciertas cepas a condiciones adversas ha dado lugar a búsquedas de nuevos microorganismos que tengan dicho potencial. $A$ nivel de Latinoamérica se han realizado varios trabajos enfocados a la identificación de cepas en biopilas o material en descomposición con la proyección de producir inoculantes para mejorar la eficiencia de estos procesos (Gaitan, 2007). La heterogeneidad en los resultados luego de la aplicación de los inoculantes y la necesidad de obtener mejores rendimientos además de mejorar las metodologías sugiere la posibilidad de buscar cepas robustas específicas.

Las nuevas indagaciones genómicas arrojan resultados sorprendentes e inesperados al identificar nuevas cepas con un alto potencial de degradación como es el caso del hongo patógeno de plantas Ustilago maydis (Couturier et al., 2012). De igual forma el estudio llevado a cabo por el grupo de investigación de Cadete et al (2012), descubrió la presencia de cepas de levaduras fermentadoras de D-xilosa, en los bosques amazónicos del Brasil, que pueden tener un uso prometedor en la producción de etanol o xilitol a partir de hidrolizados hemicelulósicos de bagazo de caña de azúcar.

Gracias a la ingeniería genética grupos de investigación a nivel mundial, también han iniciado proyectos de transformación genética de cepas para su uso en biorreactores. El ascomicete Hypocrea jecorina (Trichoderma reesei) ha sido ampliamente estudiado, la creación de mutantes para su evaluación en diferentes fuentes de carbono es una estrategia que ha permitido la evaluación del incremento de la producción de celulasa para correlacionarla con genes específicos (Dashtban et al., 2011).

Existen varias perspectivas en relación a nuevas enzimas fúngicas de gran potencial para superar el reto de los sustratos recalcitrantes, entre estas se destacan la regulación fisiológica de las enzimas, uso de nuevos inductores para mejorar la producción, uso de múltiples cepas microbianas o mezclas de enzimas, clonación de genes para monitorear nuevas generaciones de enzimas y aplicaciones de nanobiotecnología en enzimología (Godliving, 2012). 


\section{Bibliografía}

Arvanitoyannis I. and Varzakas T. (2008). Vegetable Waste Treatment: comparison and critical presentation of methodologies. Critical Reviews in Food Science and Nutrition, Volume 48, Issue 3, $205-247$.

Barrera L., Charry N. (2008). Producción y evaluación de un inoculante microbiano con capacidad amilolítica a partir de un proceso de compostaje de residuos de lechuga. Pontificia Universidad Javeriana. Microbiología Industrial. Bogotá - Colombia.

Benitez S. \& Gonzales L. (2003). Aceleración del proceso de compostaje utilizando Azotobacter, Azospirillum, Lactobacillus, Saccharomyces y Trichoderma harianum. Pontificia Universidad Católica del Ecuador. Microbiología.

Cariello M, Castañeda L, Riobo I, González J. (2007). Inoculante de microorganismos endógenos para acelerar el proceso de compostaje de residuos sólidos urbanos. R.C. Suelo Nutr. Veg. 7 (3).

Chefetz, B., Hatcher, P.G., Hadar, Y., Chen, Y. (1996). Chemical and biological characterization of organic matter during composting of municipal solid waste. Journal of Environmental Quality 25, 776-785.

Coscollá Ramón. (2008). Residuos de productos fitosantiarios. Generalitat valenciana. Dirección general de investigación y tecnología agroalimentaria. Area de protección agroalimentaria.

Couturier M, Navarro D, Olivé C, Chevret D, Haon M, Favel A, Lesage L, Henrissat B, Coutinho P, Berrin J. (2012). Post-genomic analyses of fungal lignocellulosic biomass degradation reveal the unexpected potential of the plant pathogen Ustilado maydis. BMC Genomics 13: 57.

Dashtban M, Buchkowski R, Qin W. (2011). Effect of different carbon sources on cellulose production by Hypocrea jecorina (Trichoderma reesei) strains.

Dashtban M., Schraft H., Syed T., Qin W. (2010). Fungal biodegradation and enzymatic modification of lignin. Int J Biochem Mold Biol. 1 (1): $36-50$.

Dashtban M., Schraft H., Wensheng Q. (2009). Fungal Bioconversion of Lignocellulosic residues; opportunities and perspectives. Int J. Biol.Sci; 5 (6): 576-595.

Doddapaneni H., Chakraborty R. and Yadav J. (2005). Genome-wide structural and evolutionary analysis of the P450 monooxygenase genes (P450ome) in the white rot fungus Phanerochaete chrysosporium: Evidence for gene duplications and extensive gene clustering. BMC Genomics, 6:92. 
Elisashvili V., Kachlishvili E., Penninckx M. (2008). Effect of growth substrate, method of fermentation, and nitrogen source on ligno cellulose-degrading enzymes production by whiterot basidiomycetes. J Ind. Microbiol Biotechnol. 35:1531-1538.

Eweis J., Ergas J., Chag D \& Schoroeder E. (1999). Principios de biorrecuperación. España. Mac Graw Hill.

Forgaty Andrew and Olli Tuovinen. (1991). Microbiological degradation of pesticides in yard waste composting. Microbiological Reviews. Vol 55. No. 2. P $225-233$.

Gaitan D. y Perez L. (2007). Aislamiento y evaluación de microorganismos celulolíticos a partir de residuos vegetales frescos y en compost generados en un cultivo de crisantemo (Dendranthema grandiflora). Pontificia Universidad Javeriana. Colombia.

Godliving Y. (2012). Lignocellulolytic enzymes from tropical fungi: Types, substrates and applications. Scientific Research and assays. Vol 7 (15) pp 1544 - 1555.

González R. (2007). Presencia de residuos de fungicidas e insecticidas en muestras comerciales de hortalizas de hoja. Tesis de licenciatura.

Grijalva N. (2007). Evaluación de un inoculante termofílico como acelerador del proceso de compostaje de residuos vegetales de la Florícola Jardines Piaverí “. Escuela Politécnica del Ejército. Ingeniería en Biotecnología.

Gupta P, Samant K, Sahu A. (2011). Isolation of cellulose - degrading bacteria and determination of their cellulolytic potencial. International Journal of Microbiology.

Himmel ME, Ding SY, Johnson DK, Adney WS, Nimlos MR, Brady JW, Foust TD. (2007) Biomass recalcitrance: engineering plants and enzymes for biofuels production. Science; 315:804807.

Huang D., Zeng G., Feng C., Hu S., Zhao M., Lai C., Zhang Y., Jiang X., Liu H. (2010). Mycelial growth and solid-state fermentation of lignocellulosic waste by white-rot fungus Phanerochaete chrysosporium under lead stress. Chemosphere 81: 1091 - 1097.

Kumar R. \& Shweta. (2011). Enhance of wood waste decomposition by microbial inoculation prior to vermicomposting. Bio resource technology 102: 1475-1480.

Lee C., Darah I., Ibrahim C. (2010). Production and Optimization of cellulase enzyme using Aspergillus niger USM Al 1 and comparison with Trichoderma reesei via solid state fermentation system. Biotechnology Research International.

Li L., Li XZ., Tang WZ, Zhao J., Qu YB. (2008). Screening of a fungus capable of powerful and selective delignification on wheat straw. Lett. Appl. Microbiol. 47(5): 415 -20. 
Ljungdahl LG. (2008) The cellulase/hemicellulase system of the anaerobic fungus Orpinomyces PC-2 and aspects of its applied use. Ann N Y Acad Sci; 1125:308-321.

Lu WJ, Wang HT, Nie YF, Wang ZC, Huang DY, Qiu XY, Chen JC. (2004). Effect of inoculating flower stalks and vegetable waste with ligno-cellulolytic microorganisms on the composting process. J Environ Sci Health B. 39(5-6):871-87.

Lugo, S. y Gitscher, H. (2005) Evaluación de los proyectos de compostaje en el Ecuador. Fundación Natura - Repamar - Cepis-GTZ. Quito.

Lynd L., Van Zyl W., McBride J., and Laser M. (2005). Consolidated bioprocessing of cellulosic biomass: an update. Current opinion in biotechnology 16:577-583.

Lynd LR, Weimer PJ, Van Zyl WH. (2002) Pretorius IS: Microbial cellulose utilization: fundamentals and biotechnology. Microbiol. Mol. Biol. Rev.; 66:506-577.

Martinez A., Speranza M., Ruiz F., Ferreira P., Camarero S., Guillen F., Martinez M., Gutierrez A., Del Río J. (2005). Research Review: Biodegradation of lignocellulosic: microbial, chemical, and enzymatic aspects of the fungal attack of lignin. International Microbiology 8: 195-204.

Oleas Nicolás, Fernández Mariana. (2001) Plaguicidas persistentes. Congreso "Implementación del Convenio de Contaminantes orgánicos persistentes".

Peng G., Zhu W., Wang H., Lü Y., Wang X., Zheng D., Cui Z. (2010). Functional characteristics and diversity of a novel lignocelluloses degrading composite microbial system with high xylanase activity. J. Microbiol. Biotechnol. 20(2):254-64.

Pereira L., Hiibel S., Prieto M., Reardon K., Pruden A. (2010) Detection an Quantification of Funtional Genes of Cellulose - degrading, fermentative, and sulfate - reducing bacteria and Methanogenic Archaea. Applied and environmental microbiology. Vol 76, No. 7, p 2192 2202.

Pérez J., Muñoz-Dorado J., De la Rubia T., Martinez J. (2002). Biodegradation and biological treatments of cellulose, hemicellulose and lignin: an overview. Int Microbial; 5:53-63.

Sánchez Carmen. (2008). Review Lignocellulosic residues: Biodegradation an bioconversion by fungi. Biotechnology Advances 27:185 - 194.

Schuster A. \& Schmol Monika. (2010). Mini-Review: Biology and biotechnology of Trichoderma. Appl. Microbiol. Biotechnol 87: 787-799.

Sharma K, Kuhad R. (2010). Genetic transformation of lignin degrading fungi facilitated by Agrobacterium tumefaciens. BMC Biotechnology 10:67. 
Taube J., Vorkamp K., Förster M., Herrmann R. (2002). Pesticide residues in Biological waste. Chemosphere 49: 1357 - 1365.

Vargas-García M.C., Suárez-Estrella F., López M.J., Moreno J.. (2007). Effect of inoculation in composting processes: Modifications in lignocellulosic fraction. Waste Management 27 1099-1107.

Yang S, Kataeva I, Hamilton S, Engle N, Tschplinski T, Doeppke C, Davis M., Westpheling J. Adams M. (2009) Efficient Degrading of Lignocellulosic plant biomass, without pretreatment, by the thermophilic anaerobe "Anaerocellum thermophilum" DSM 6725. Applied and environmental microbiology. P. 4762 - 4769

Yin ZW, Fan BQ, Ren P. (2011).Isolation and identification of a cellulose degrading fungus Y5 and its capability of degradation wheat straw. Huang Jing Ke Xue 32(1):247-52.

Zhou S, Ingram LO. (2000). Synergistic hydrolysis of carboxymethyl cellulose and acid-swollen cellulose by two endoglucanases (CelZ and CelY) from Erwinia chrysanthemi. J Bacteriol.182:5676-5682. 